\title{
Ice and ocean
}

\author{
A substantial amount of the Earth's surface water moves between ice sheets and oceans as the climate \\ oscillates on geological timescales. Ocean warming, as well as atmospheric temperature rise, affects the \\ current redistribution in response to climate change.
}

The long-term fate of coastal communities around the globe could depend on the state of the high-latitude oceans. Mounting evidence suggests that the stability of the Greenland and Antarctic ice sheets, and hence sea level, is sensitive to changes in ocean temperature. In particular, warming at intermediate depths, which reaches the underbelly of the ice, has a large potential to erode the marine glacier termini and floating ice shelves that currently buttress the larger ice masses on land.

Two articles in this issue (page 519 and page 524) explore ocean warming in relation to the ice sheets. An analysis of observations underneath a floating ice shelf off West Antarctica leads to the conclusion that ocean warming and a more vigorous circulation were responsible for accelerating melt rates; a second study projects further rises in ocean temperatures at depths of 200-500 $\mathrm{m}$ for the twenty-first century that are particularly fast near the Greenland ice sheet but still larger than last century's warming off Antarctica. The studies are complemented by a Review article (page 506), concluding that the West Antarctic ice sheet is likely to remain largely intact over the coming century, but the possibility of future collapse cannot be excluded.

Looking over past publications on the topic of the waning ice sheets, the picture that emerges is one of a steep learning curve: scientific understanding of sea-level rise has evolved considerably in the past decade or so.
When the third assessment report of the Intergovernmental Panel on Climate Change was published ten years ago, much of the focus was placed on the expected thermal expansion of the ocean, the increase in volume that is directly attributable to rising water temperatures. Since then, rapid loss of ice mass in both Greenland and Antarctica has shifted attention to gains in ocean mass, as water is transferred from the land-based ice reservoirs into the oceans.

The factors responsible for ice-sheet mass loss have come into sharper focus, too. The observed acceleration of the steady flow of ice from the large ice sheets into the sea was initially attributed to rising water pressure at the base of the ice sheet. Water generated through melting at the ice surface percolates downwards through crevasses and conduits in the ice, eventually lubricating the base of the ice sheet or glacier (Nature Geosci. 1, 297-304; 2008). But in addition, it turns out that ice loss is also induced by ocean warming. Relatively warm ocean water can eat away at the deeper parts of Greenland's marine-terminating glaciers and Antarctica's ice shelves (see, for example, http://www. nature.com/ngeo/focus/warm-ice-sheets/ index.html). The process removes some of the buttressing of the land-based ice, and can lead to thinning and retreat by means of an accelerated flow of ice into the ocean.

Of the three largest ice reservoirs on Earth - the ice sheets in Greenland, West Antarctica and East Antarctica - the West
Antarctic ice sheet is most vulnerable to warming waters. The Greenland and East Antarctic ice sheets are situated on relatively high ground, and will eventually be isolated from oceanic warmth if thinning and retreat continues. By contrast, much of West Antarctica's ice mass rests on rock below sea level, and will not be removed from the destructive influence of a warm ocean simply by retreat. Here, ocean warming at medium depths could have a substantial influence on the ultimate fate of the ice sheet.

By unlucky coincidence, there are indications that intermediate-depth ocean warming near West Antarctica may also be a particularly persistent aspect of long-term climate change. A long-term climate model projection suggests that, even if carbon dioxide emissions are eliminated entirely by the year 2100, and global mean temperatures consequently stabilize, the Southern Ocean could continue to warm for thousands of years (Nature Geosci. 4, 83-87; 2011).

Most likely, catastrophic change is not imminent, and it will take several hundred years before a substantial fraction of the ice is lost by subsurface ocean warming. But a rise in intermediate-depth ocean temperatures will be virtually impossible to counter by geoengineering, should the need arise. It would be prudent to keep a close eye on ocean temperatures near the ice sheets although the Southern Ocean with its harsh climate is one of the most difficult places in the world's oceans to monitor.

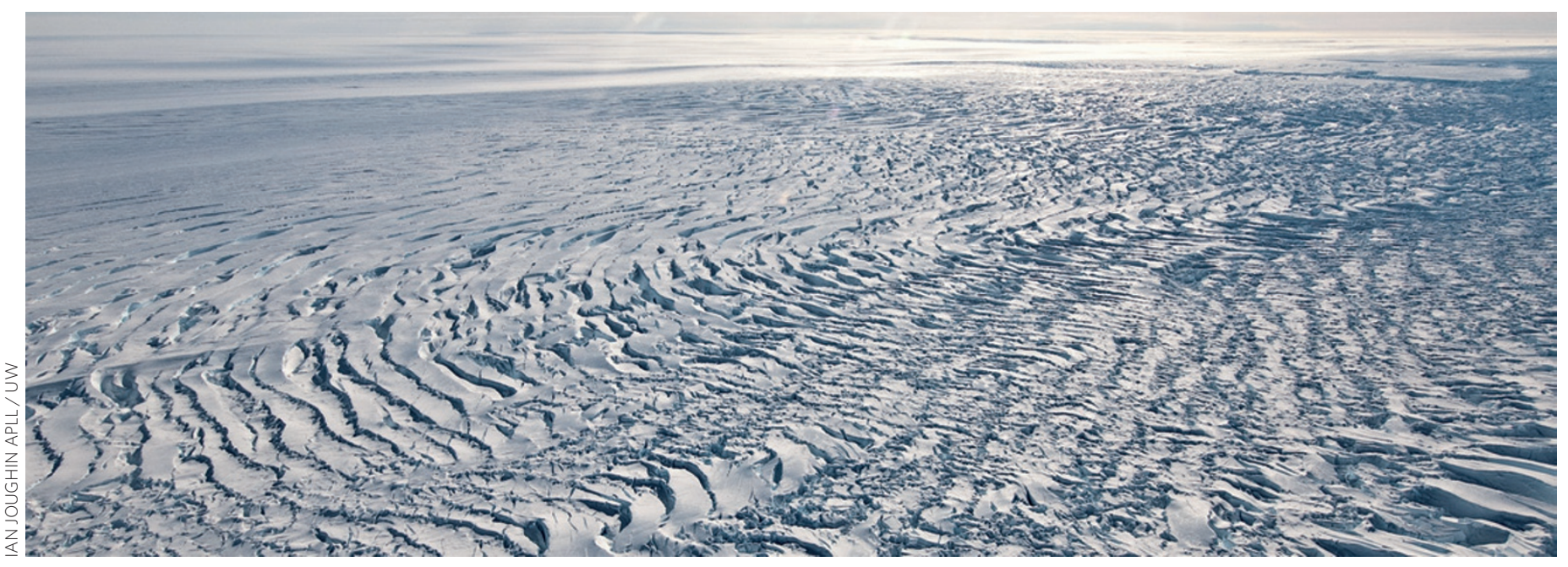

